\title{
Trichostatin A reduces cisplatin-induced ototoxicity through the STAT6 signaling pathway
}

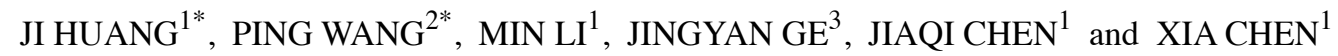 \\ ${ }^{1}$ Department of Pharmacology, College of Basic Medical Sciences, Jilin University; \\ ${ }^{2}$ Department of Otolaryngology-Head and Neck Surgery, The First Hospital of Jilin University; \\ ${ }^{3}$ Department of Physiology, College of Basic Medical Sciences, Jilin University, Changchun, Jilin, P.R. China
}

Received March 1, 2015; Accepted June 8, 2015

DOI: $10.3892 /$ ijmm.2015.2249

\begin{abstract}
Cisplatin-induced ototoxicity limits its wide application in the treatment of cancer. A number of pro-inflammatory factors have been shown to be involved in cisplatin-induced ototoxicity. Trichostatin A (TSA) is an anti-inflammatory agent that has been shown to exert protective effects against cisplatin-induced ototoxicity. In the present study, we hypothesized that TSA may protect cochlear hair cells from cisplatin-induced damage by regulating the interleukin (IL)-4/ signal transducer and activator of transcription (STAT)6 signaling pathway. Wistar rat cochlear explants were cultured in DMEM. The differentially expressed genes of the basilar membrane were identified by microarray analysis of global expression profiles. Hair cells were stained with rhodamine phalloidin and observed under a scanning electron microscope to evaluate the protective effects of TSA against cisplatininduced cochlear hair cell damage. The levels of cytokines in the supernatant of the cultured basilar membranes was measured using ELISA. STAT6 and phosphorylated (p-) STAT6 expression was measured by western blot analysis. Morphological observation revealed that cisplatin induced the disarrangement of the cochlear hair cells, as well as the fusion and detachment of the cilia, while these aberrant alterations were inhibited by TSA, suggesting that TSA exerts a protective effect against cisplatin-induced damage to hair cells. Furthermore, the increase in the expression of STAT6 and p-STAT6 induced by cisplatin was reversed by treatment with TSA, accompanied by the decreased expression of IL-1 $\beta$, IL-4 and IL-6. Therefore, our data demonstrate that TSA reduces cisplatin-induced ototoxicity by inhibiting pro-inflammatory factor-mediated STAT6 signaling. Thus, TSA may be used to
\end{abstract}

Correspondence to: Professor Xia Chen, Department of Pharmacology, College of Basic Medical Sciences, Jilin University, 126 Xinmin Street, Changchun, Jilin 130021, P.R. China

E-mail: chenx6166@163.com

*Contributed equally

Key words: trichostatin A, ototoxicity, signal transducer and activator of transcription 6, inflammation prevent the side-effects associated with the use of cisplatin in cancer treatment.

\section{Introduction}

Cisplatin is widely used as an effective chemotherapeutic drug in the clinical treatment of lung, head and neck and bladder cancer (1). However, side-effects, such as ototoxicity, nephrotoxicity and neurotoxicity, limit its wide application in clinical practice $(2,3)$. As regards ototoxicity, cisplatin induces the loss of cochlear hair cells, the degradation of the stria vascularis and a reduction in the number of spiral ganglion cells, which ultimately results in high frequency hearing loss in both ears $(4,5)$. The mechanisms underlying cisplatin-induced ototoxicity have not been fully elucidate to date. It has been demonstrated that the increased production of pro-inflammatory factors, such as tumor necrosis factor- $\alpha$ (TNF- $\alpha$ ), interleukin (IL)-1 $\beta$ and IL-6 is involved in cisplatin-induced ototoxicity in cochlear hair cells (6). The production of these pro-inflammatory factors is regulated by the signal transducer and activator of transcription (STAT) proteins (7).

STAT proteins are important transcription factors. They promote signal conveying between cytokines and their receptors to induce the transcription of downstream target genes (8). Activated STATs mediate a variety of biological processes, such as cell development, proliferation, differentiation, migration and apoptosis, as well as the response to different stimuli in cells. Therefore, the STAT family is closely associated with a variety of diseases $(9,10)$. STAT6, one of the crucial members of the STAT family, plays a key role in cisplatin-induced ototoxicity. During the development of ototoxicity induced by cisplatin, STAT6 is activated by the binding of IL-4 to its receptor (11), and then conveys signals to the nucleus to promote the transcription of downstream genes which are associated with inflammatory responses. The IL-4/STAT6 signaling pathway is associated with cisplatin-induced ototoxicity (11).

Trichostatin A (TSA) is an anti-inflammatory agent derived from the metabolites of streptavidin (12). It has been shown that TSA protects cochlear hair cells from aminoglycoside antibioticsinduced cell death, thereby exhibiting anti-ototoxic acitivity (13). Coincidentally, the ototoxicity caused by cisplatin is similar to that induced by aminoglycoside antibiotics; thus, it is conjectured that TSA may inhibit cisplatin-induced ototoxicity as well (14). 
This conjecture was confirmed by a study in which cisplatininduced auditory cell toxicity was successfully inhibited by treatment with $200 \mathrm{nM}$ TSA (15). Although the protective mechanisms of TSA against cisplatin-induced ototoxicity are largely unclear, the implications of its anti-inflammatory responses have been demonstrated in a number of previous studies $(16,17)$. In the present study, we hypothesized that TSA may protect cochlear hair cells from cisplatin-induced damage by regulating the IL-4/ STAT6 signaling pathway which is associated with inflammatory responses, and we succeeded in confirming this hypothesis.

\section{Materials and methods}

Animals. Wistar rats (newborn 3-4 days old) were provided by the Animal Center of Jilin University [SCXK (JI) 2007-0003]. All experimental procedures were approved by the Animal Ethics Committee of Jilin University, Changchun, China.

Reagents. TSA, cisplatin and rhodamine phalloidin were purchased from Sigma (St. Louis, MO, USA). Dulbecco's modified Eagle's medium (DMEM) and fetal bovine serum (FBS) were provided by Invitrogen (Carlsbad, CA, USA). The IL-1 $\beta$, IL-4 and IL-6 enzyme-linked immunosorbent assay (ELISA) kits were obtained from Wuhan Boster Biological Engineering Co., Ltd. (Wuhan, China). Rabbit polyclonal antibody to STAT6 (sc-981) was obtained from Santa Cruz Biotechnology, Inc. (Santa Cruz, CA, USA). Mouse monoclonal anti-phosphorylated (p-)STAT6 antibody (05-590) was purchased from Millipore (Bedford, MA, USA).

Gene microarray and data analysis. To identify the differentially expressed genes (DEGs), we used our previous microarray based on cochlear explants treated for $6 \mathrm{~h}$ with cisplatin or cisplatin plus TSA compared with cochlear explants without drug treatment (15). We calculated the DEGs between the control and cisplatin-treated explants, as well as between the cisplatinand cisplatin plus TSA-treated explants. The fold change values were calculated. The genes with a fold change in expression of $>2$ or $<0.5$ were selected as the DEGs. Finally, to implement subpathway enrichment, we matched the DEGs of the Wistar rats with the corresponding orthologous human genes. A total of 467 differentially expressed orthologous human genes were obtained between the control samples and those treated with cisplatin. A total of 2,588 differentially expressed orthologous human genes were obtained between the samples treated with cisplatin alone and those treated with cisplatin plus TSA.

Identification of subpathways using Subpathway Miner. To identify the perturbed subpathways following treatment with cisplatin alone or with cisplatin plus TSA, we used the Subpathway Miner software package which is a flexible subpathway identification method (18). With this software, the pathway structure data of KEGG are converted to R undirected graph objects. The enzymes are then considered as nodes and 2 nodes are connected by an edge if they are in the same reactions. This software package can transform the entire pathway into subpathways using the 'k-clique' method, which is defined as a subgraph in which the distance between any 2 nodes is no greater than a parameter $\mathrm{k}$. In this study, the value of this parameter was set as $\mathrm{k}=3$.
We then imported 2 sets of DEGs (control vs. cisplatin; cisplatin vs. cisplatin plus TSA) into Subpathway Miner and this software identified 2 sets of significantly enriched subpathways. A value of $\mathrm{P}<0.01$ was selected as the cut-off criterion for the statistically significant subpathways.

Culture of basilar membrane. The culture of the basilar membrane was performed as previously described (19). The rats were sacrificed by decapitation after being cleaned with $75 \%$ ethanol, and the parietal bone was opened and the brain tissue was removed. The otic vesicles were removed and placed into cold D-Hank's solution without $\mathrm{Ca}^{2+}$ and $\mathrm{Mg}^{2+}$. The connective tissues around the spiral case was carefully removed under an anatomical microscope, and the spiral case was opened and the membranous labyrinth was completely exposed. The modiolus, spiral ligament, vestibular membrane and tectorial membrane were carefully dissected, leaving the whole basilar membrane with the organ of Corti and spiral ganglion neurons. Briefly, the entire basilar membrane was placed on a $35 \mathrm{~mm}$ culture dish with the convex side up and incubated with $1 \mathrm{ml}$ of DMEM supplemented with $10 \% \mathrm{FBS}$ in an incubator containing $5 \% \mathrm{CO}_{2}$ at $37^{\circ} \mathrm{C}$ for $24 \mathrm{~h}$. The culture medium was then replaced with medium supplemented with TSA (at final concentrations of 50, $100,200$ or $300 \mathrm{nM})$, cisplatin $(150 \mu \mathrm{M})$, or a combination of TSA and cisplatin ( $150 \mu \mathrm{M}$ cisplatin plus TSA $200 \mathrm{nM})$, followed by a further $24 \mathrm{~h}$ of incubation. The samples incubated with drug-free medium were considered as the controls. Each experiment was repeated at least 3 times.

Staining of hair cells. At the end of the incubation period, the cultures were fixed with $4 \%$ paraformaldehyde for $30 \mathrm{~min}$ and washed 3 times with $0.1 \mathrm{mM}$ phosphate-buffered saline (PBS). They were then incubated in $0.25 \%$ Triton X-100 for $5 \mathrm{~min}$. The cultures were then incubated with rhodamine phalloidin in the dark at room temperature for approximately $30 \mathrm{~min}$. The cultures were observed under a laser confocal microscope (FluoView FV1000, serial no. 08004045; Olympus, Tokyo, Japan) and the images were captured using FV10-ASW 1.7 Viewer software (FluoView FV1000; Olympus). The number of hair cells per $0.24 \mathrm{~mm}$ length of the cochlea was counted from the apical back to the basal turn.

Scanning electron microscopy of hair cells. The specimens were prepared according to methods described by Segawa et al (20). The basilar membranes were washed with PBS and fixed with $2.5 \%$ glutaraldehyde for $24 \mathrm{~h}$ and then further fixed with $1 \%$ osmium tetroxide $\left(\mathrm{OsO}_{4}\right)$ for $2 \mathrm{~h}$. The specimens were dehydrated in a graded series of ethanol $(50,70,80,90$ and $100 \%$, $10 \mathrm{~min}$ each) and then in tert-butyl alcohol for $10 \mathrm{~min}$. After drying with a carbon dioxide critical point dryer, the specimens were attached to aluminum stubs and coated with Au by an ion sputtering coating machine. Finally, a Hitachi scanning electron microscope (S-3400N, serial no. 09008178; Hitachi High Technologies, Tokyo, Japan) was employed to examine the surface of the basilar membranes and capture images.

ELISA. The specimens were grouped according to the treatment concentrations of TSA or cisplatin, 15 per group, as follows: i) control group (not treated with drugs); ii) group treated with $150 \mu \mathrm{M}$ cisplatin; iii) group treated with $200 \mathrm{nM}$ TSA; 
A

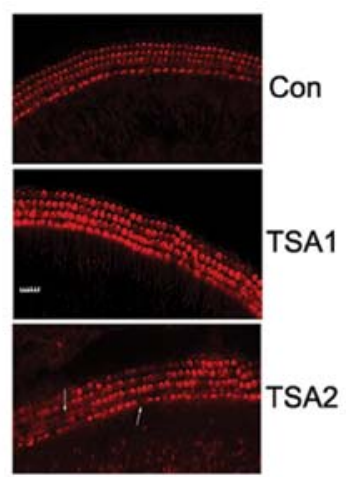

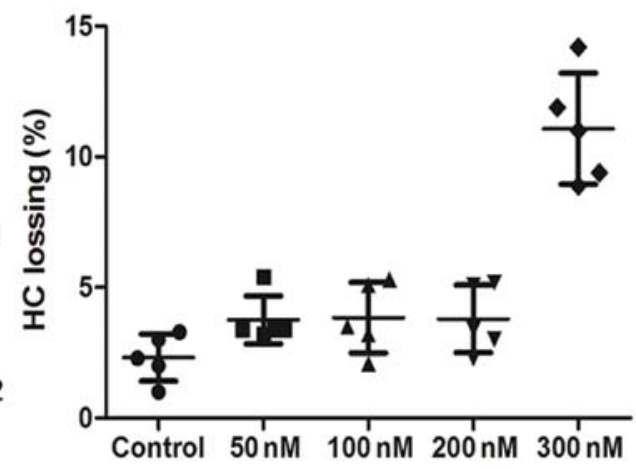

B
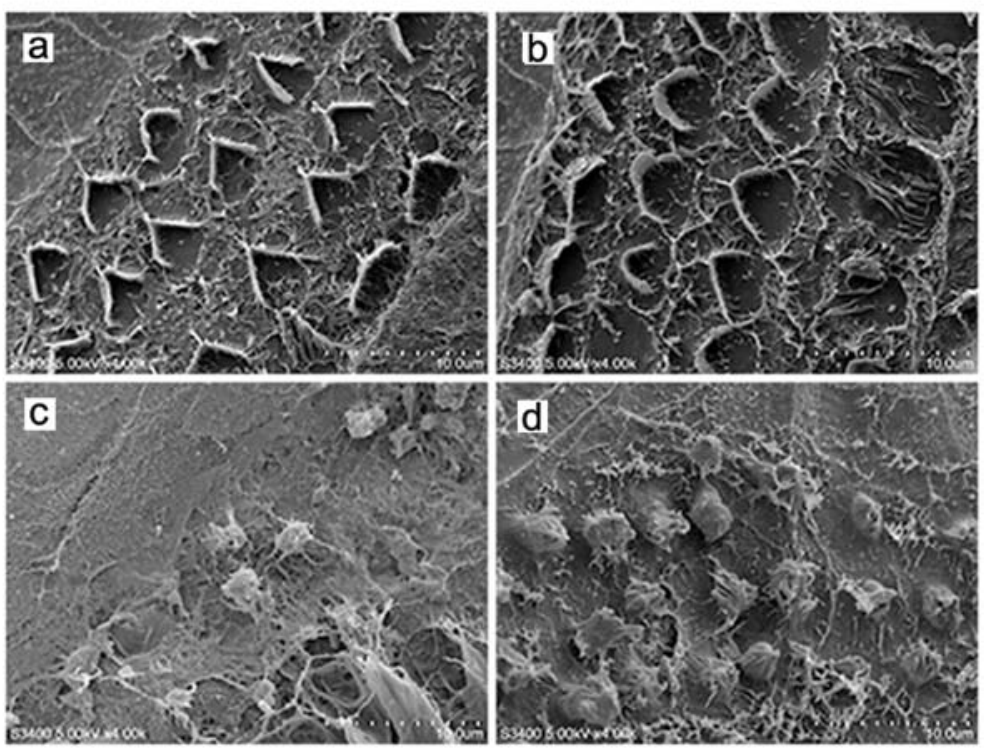

Figure 1. Effect of trichostatin A (TSA) on the survival of cochlear hair cells in vitro. (A) The results of cytochemical staining revealed that 24 h after the administration of TSA, TSA at $200 \mathrm{nM}$ had no effect on the survival of cochlear hair cells, while TSA at $300 \mathrm{nM}$ induced hair cell loss. Quantitative analysis of hair cells in the middle turn of the cochlea revealed that the rate of the loss of hair cells following treatment with $300 \mathrm{nM}$ TSA was approximately $11.8 \%$, which was significantly higher than that following treatment with TSA at $200 \mathrm{nM}(\mathrm{P}<0.001)$. Scale bar, $20 \mu \mathrm{M}$. (B) Treatment with TSA at $200 \mathrm{nM}$ protected the cochlear hair cells from the damage induced by cisplatin. (a) Basilar membrane cultured in $10 \%$ FBS-DMEM; (b) $200 \mathrm{nM}$ TSA for 24 h; (c) $150 \mu \mathrm{M}$ cisplatin for $24 \mathrm{~h}$; (d) $200 \mathrm{nM}$ TSA for $1 \mathrm{~h}$, followed by the administration of cisplatin of $150 \mu \mathrm{M}$ for $24 \mathrm{~h}$.

and iv) group treated with cisplatin $150 \mu \mathrm{M}+$ TSA $200 \mathrm{nM}$. Following treatment for $24 \mathrm{~h}$, the levels of cytokines in the supernatant of the cultured basilar membranes were measured using commercial two-site rat IL-4, IL-6 and IL-1 $\beta$ ELISA kits according to the manufacturer's instructions, respectively. The absorbance was detected at $450 \mathrm{~nm}$ to evaluate the levels of IL-4, IL-6 and IL-1 $\beta$ at different time points following treatment.

Western blot analysis. Western blot analysis was performed as previously described (11). Total proteins from the basilar membranes were extracted with RIPA-containing phenylmethanesulfonylfluoride and protein phosphatase inhibitor. The concentrations of proteins were determined using BCA protein assay kits, after which proteins were separated by $8 \%$ sodium dodecyl sulfate-polyacrylamide gel electrophoresis (SDS-PAGE) and transferred onto polyvinylidene difluoride (PVDF) membranes. After washing with $1 \mathrm{X}$ Tris-buffered saline/ Tween-20 (TBST), the PVDF membranes were stained in Ponceau $S$ staining solution on a shaking table at low velocity for 3-5 min, followed by blocking in $1 \mathrm{X}$ TBST including $5 \%$ skim milk for $2 \mathrm{~h}$ at room temperature or overnight at $4^{\circ} \mathrm{C}$. The PVDF membranes were probed with primary antibodies $(1: 1,000)$ with gentle shaking at room temperature for $2 \mathrm{~h}$ and then at $4^{\circ} \mathrm{C}$ overnight. Subsequently, the membranes was washed with $1 \mathrm{X}$ TBST and incubated with corresponding horseradish peroxidase-conjugated secondary antibodies $(1: 1,000)$ for $2 \mathrm{~h}$ at room temperature. Finally, the protein bands were identified by enhanced chemiluminescence kits. Image J 1.41 software (National Institutes of Health, Bethesda, MD, USA) was used to analyze the optical density of the bands. $\beta$-actin was used as a normalizing protein.

Statistical analysis. The data were analyzed utilizing SPSS 17.0 software. All results are expressed as the means \pm SD. The analysis of hair cell loss was performed by one-way analysis of variance (ANOVA). The difference between any 2 groups was determined by the $\mathrm{Q}$ test. A value of $\mathrm{P}<0.05$ was considered to indicate a statistically significant difference.

\section{Results}

Hair cell survival in the basilar membrane following treatment with TSA. Immunocytochemical staining revealed that the hair 


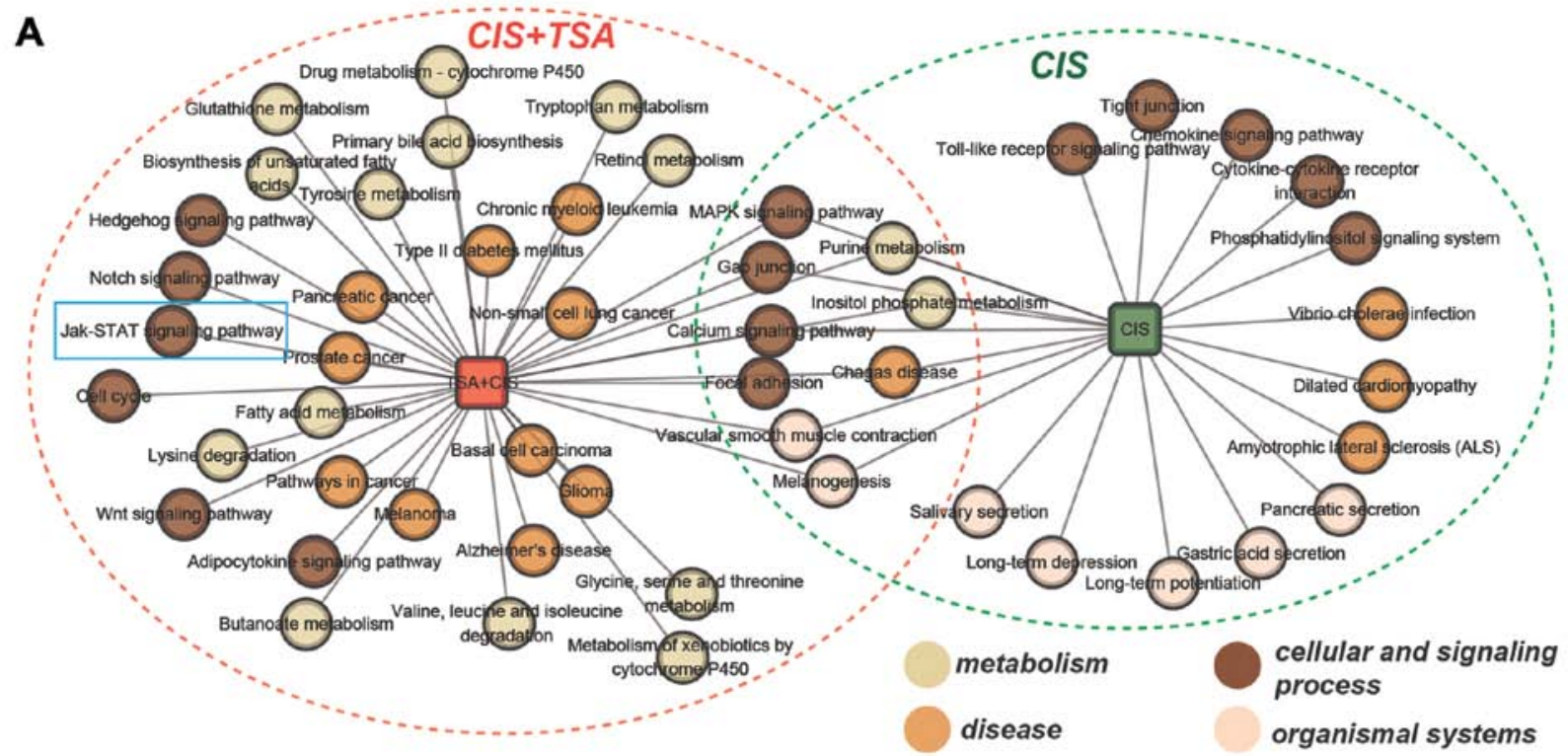

B

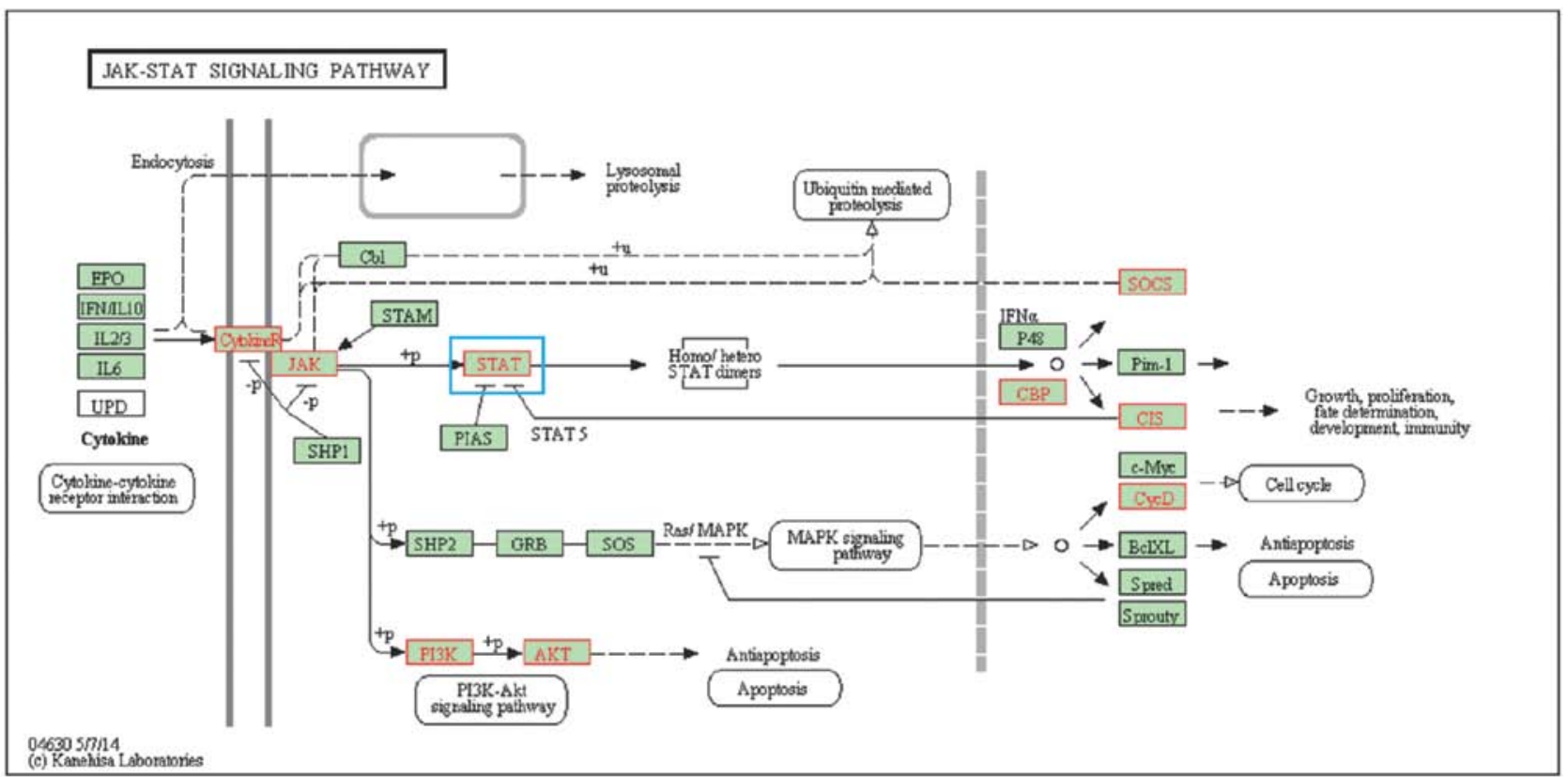

Figure 2. Results of subpathway enrichment analysis. (A) Perturbed pathways under conditions of treatment with cisplatin (CIS) and treatment with CIS plus trichostatin A (TSA). The blue rectangle represents the JAK-STAT signaling pathway. (B) Differentially expressed genes (DEGs) in the JAK-STAT signaling pathway. The blue rectangle represents STAT.

cells of the basilar membrane grew well following culture. Three lines of cochlear outer hair cells formed an orderly ' $\mathrm{V}$ ' glyph with a clearly visible outline and the cilia of the inner hair cells aligned as a '-' glyph. The loss of a few hair cells was observed at the top back to the basal turn of the cochlea. Following treatment with TSA (50, 100 or $200 \mathrm{nM}$ ) for $24 \mathrm{~h}$, only a slight loss of outer and inner hair cells was observed. However, there was a significant increase in the loss of hair cells following treatment with TSA $300 \mathrm{nM}$ (Fig. 1A). Thus, treatment with TSA at a low concentration had no obvious effect on cochlear hair cells.

TSA reduces cisplatin-induced damage to hair cells in the basilar membrane. Scanning electron microscopy revealed that the hair cells had a normal morphology with an orderly arrangement regardless of the absence or presence of $200 \mathrm{nM}$ TSA in the medium. However, following treatment with $150 \mu \mathrm{M}$ cisplatin for $24 \mathrm{~h}$, the number of hair cells was markedly reduced and the cilia of the hair cells became disorganized, fused and detached. Treatment with a combination of $200 \mathrm{nM}$ TSA and $150 \mu \mathrm{M}$ cisplatin significantly decreased hair loss compared to treatment with cisplatin alone (Fig. 1B).

Identification of the mechanisms repsonsible for the protective effects of TSA against cisplatin-induced ototoxicity based on subpathway enrichment analysis. To elucidate the mechanisms responsible for the protective effects of TSA against cisplatininduced ototoxicity, we identified 2 sets of corresponding dysregulated subpathways using Subpathway Miner (see 
A
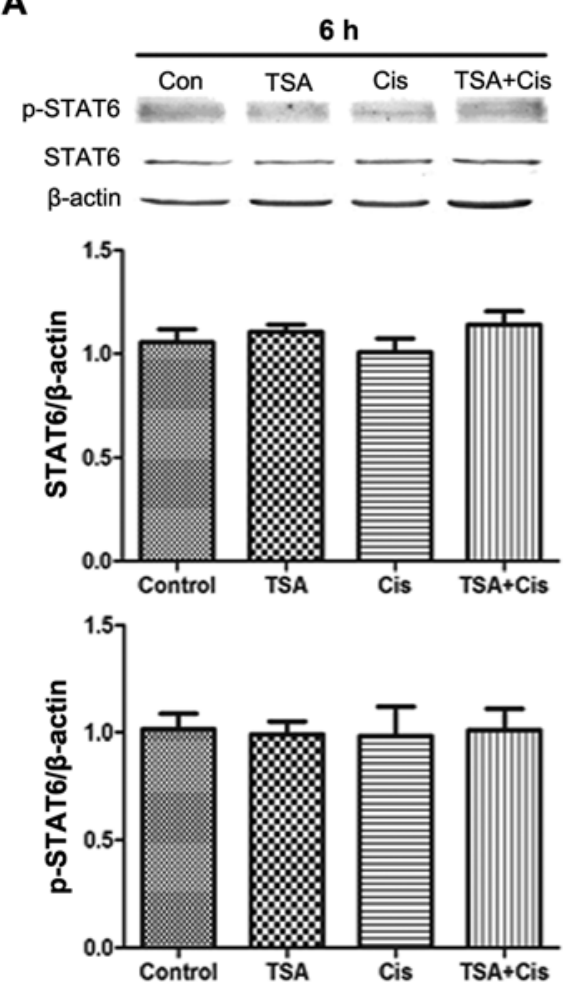

B
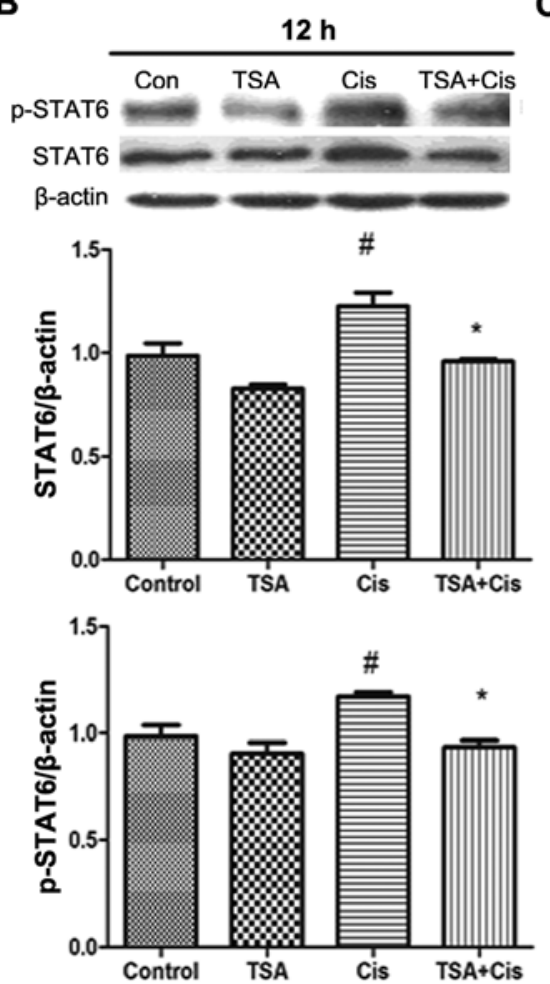

C
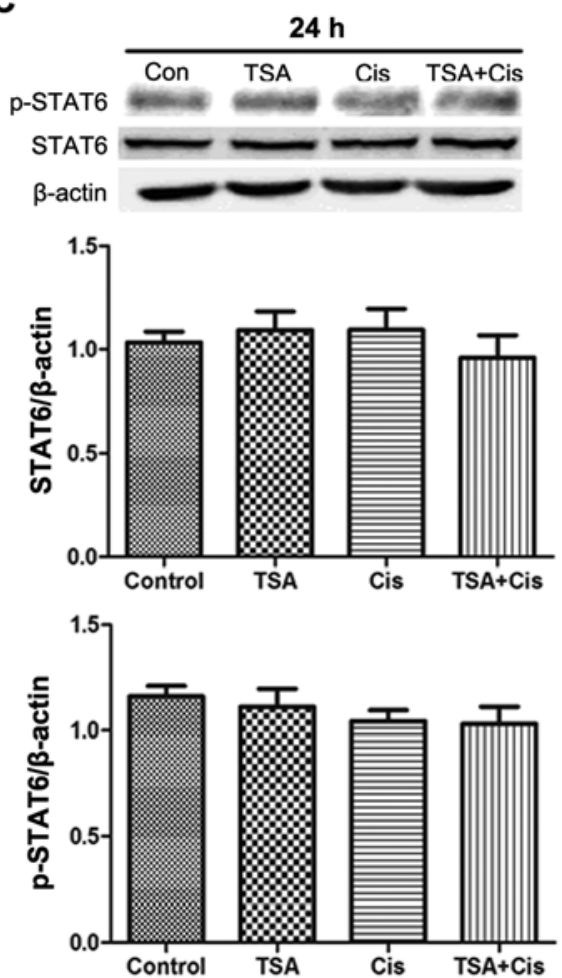

Figure 3. Expression of STAT6 in the cultured basilar membrane examined by western blot analysis. Quantitative analysis of the expression of STAT6 relative to $\beta$-actin examined by western blot analysis. (A) No significant difference was observed in the expression of p-STAT6 and STAT6 in the basilar membrane at $6 \mathrm{~h}$ among the 4 groups. (B) At $12 \mathrm{~h}$, the expression of p-STAT6 and STAT6 significantly increased in the cisplatin-treated group compared with the control. Moreover, the increase in this expression was reduced by treatment with trichostatin A (TSA) in the TSA + Cis group. (C) No change in the expression of the 2 proteins was detected at 24 h. ${ }^{\#} \mathrm{P}<0.05$ vs. control group; ${ }^{*} \mathrm{P}<0.05$ vs. Cis group. Cis, cisplatin.

Materials and methods; with a strict cut-off value of $\mathrm{P}<0.01$ ) One set corresponded to dysregulated subpathways under conditions of treatment with cisplatin alone (62 subpathways corresponding to 21 entire pathways), and the other set corresponded to dysregulated subpathways under conditions of treatment with cisplatin plus TSA compared to treatment with cisplatin alone (89 subpathways corresponding to 38 entire pathways). We then compared the 2 sets of subpathways. Fig. 2A shows a network of entire pathways under 2 treatment conditions (cisplatin alone or cisplatin plus TSA). We found that there were 8 common pathways and that cisplatin plus TSA treatment affected extra pathways compared to treatment with cisplatin alone. These extra pathways were considered to be associated with the potential molecular mechanisms responsible for the TSA-mediated protective effects against cisplatin ototoxicity (Fig. 2A). We focused on the cell signaling process. Of these pathways, the JAK-STAT signaling pathway (blue rectangle in Fig. 2A) was shown to play a pivotal role in cisplatin-mediated pro-inflammatory cytokine production and ototoxicity. In order to investigate the mechanisms responsible for the protective effects of TSA against cisplatin ototoxicity, we mapped the DEGs of cisplatin vs. cisplatin plus TSA into the JAK-STAT signaling pathway using the KEGG mapping tool. We found that some key proteins were annotated, including JAK and STAT (blue rectangle in Fig. 2B).

TSA increases the expression of STAT6 in the basement membrane. The expression levels of STAT6 in the basement membrane were measured by western blot analysis (Fig. 3).
STAT6 protein and p-STAT6 expression levels were increased following treatment with cisplatin for $12 \mathrm{~h}$. By contrast, no changes were observed in the expression of STAT6 and p-STAT6 following treatment with cisplatin for 6 and $24 \mathrm{~h}$. Furthermore, the increase in the expression of STAT6 and p-STAT6 induced by cisplatin was downregulated by treatment with TSA. These findings indicated that TSA exerts its protective effects against cisplatin-induced ototoxicity through the STAT6 pathway.

TSA decreases the expression inflammatory cytokines in the cochlea following treatment with cisplatin. The levels of IL-4, IL-1 $\beta$ and IL-6 in the supernatant of the cultured basilar membrane were measured by ELISA. The concentrations of these cytokines in the treatment groups were upregulated by cisplatin compared with the control group. The expression levels of IL-1 $\beta$ and IL- 6 reached maximum levels at $12 \mathrm{~h}$ following treatment with cisplatin. The increase in the expression levels of IL-1 $\beta$ and IL- 6 induced by cisplatin was inhibited by treatment with TSA (Fig. 4A and B). Subsequently, treatment with TSA inhibited the increase in the expression of IL-4 induced by cisplatin (Fig. 4C). Thus, the secretion of inflammatory cytokines related to STAT6 in the basilar membrane induced by cisplatin was time-dependent and was inhibited by TSA.

\section{Discussion}

The incidence of cisplatin-induced ototoxicity is high in patients who have undergone cisplatin-based chemotherapy. 

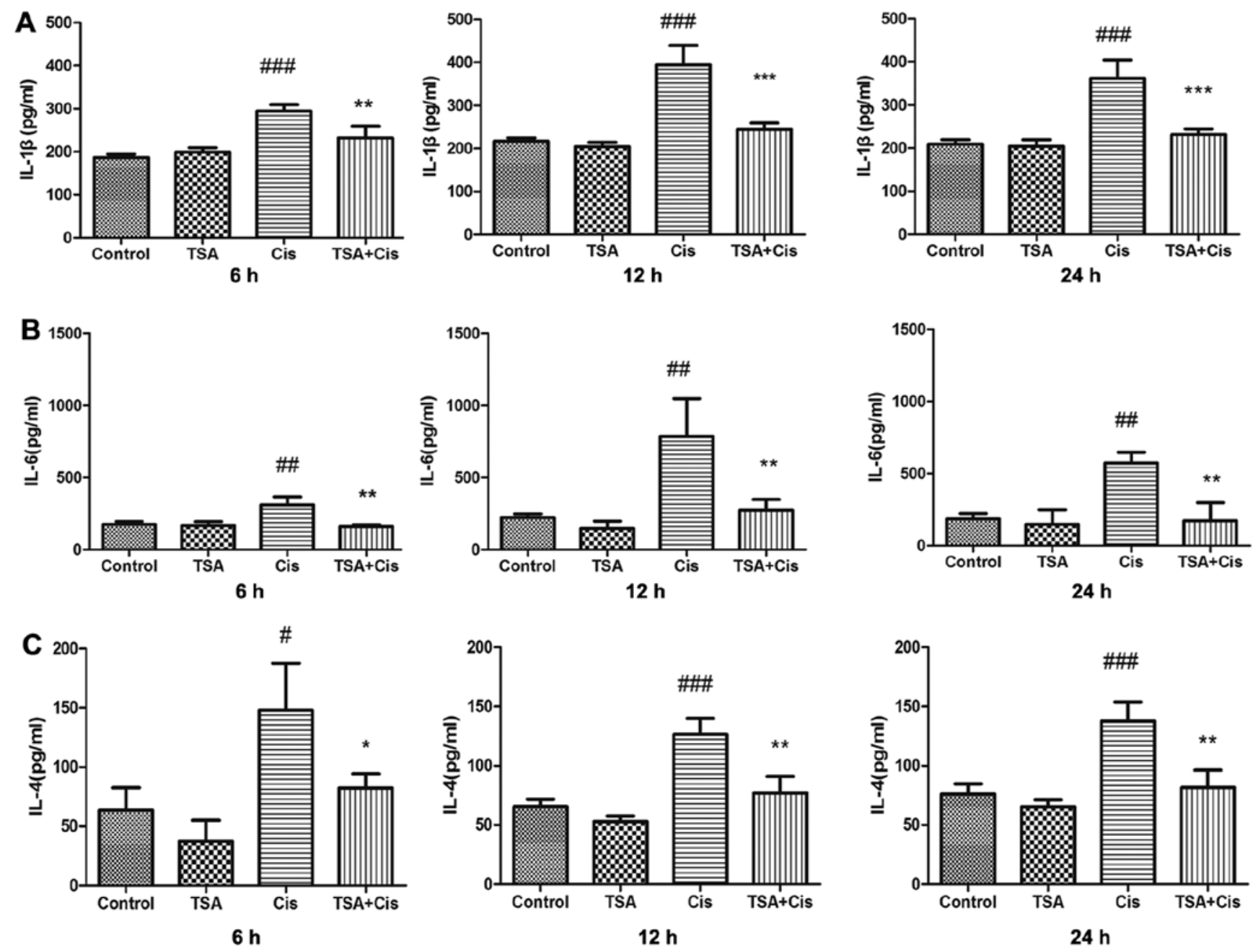

Figure 4. Detection of interleukin (IL)-1 $\beta$, IL-4, IL-6 expression in supernatant of cultured basilar membrane by ELISA. Supernatant of cultured basilar membrane was collected following treatment with trichostatin A (TSA) and/or cisplatin. TSA alone had no effect on the secretion of (A) IL-1 3 , (B) IL-6 and (C) IL-4 in the basilar membrane. Following treatment with cisplatin, the level of IL-1 $\beta$ and IL-6 increased and reached peak levels at $12 \mathrm{~h}$, and IL- 4 increased to its peak level at $6 \mathrm{~h}$. In the TSA + Cis group, treatment with TSA at the concentration of $200 \mathrm{nM}$ restored the increased level of each cytokine to a normal level. " $\mathrm{P}<0.05$ vs. control group; ${ }^{\# /} \mathrm{P}<0.01$ vs. control group; ${ }^{\# \#} \mathrm{P}<0.001$ vs. control group; ${ }^{*} \mathrm{P}<0.05$ vs. Cis group; ${ }^{* *} \mathrm{P}<0.01$ vs. Cis group; ${ }^{* * *} \mathrm{P}<0.001$ vs. Cis group. Cis, cisplatin.

The damage caused by cisplatin mainly occurs in the outer hair cells of the organ of Corti (21). In the present study, cisplatin induced hair cell loss, as well as the collapse and disorder of cultured basilar membranes. This effect was more prominent in the outer hair cells than in the inner hair cells, further confirming that cochlear hair cells are the main target of cisplatin ototoxicity. Treatment with TSA alleviated the cisplatin-induced loss of hair cells of the basilar membrane, which suggests that TSA exerts a protective effect against cisplatin-induced ototoxicity.

We identified 8 common pathways in the network of entire pathways following treatment with cisplatin alone or cisplatin plus TSA using Subpathway Miner. We focused on the role of the JAK-STAT signaling pathway in the cisplatin-mediated production of pro-inflammatory cytokines and ototoxicity. TSA has been shown to suppress the growth of colorectal cancer cells through the regulation of downstream targets of JAK2/STAT3 signaling (22). We found that some key proteins were annotated, including JAK and STAT.

Recently, more attention has been paid to the mechanisms responsible for cell damage induced by cisplatin, such as inflammation. For example, in a previous study, the protective effects of $\alpha$-lipoic acid against cisplatin-induced ototoxicity were shown to be mediated through the regulation of mitogenactivated protein kinases (MAPKs) and pro-inflammatory cytokines in cisplatin-treated HEI-OC1 cells (23). Reactive oxygen species (ROS) and inflammation are the major contributors to cisplatin-induced hearing loss. This suggests that controlling inflammation by the inhibition of STAT1-dependent pathways in the cochlea may serve as an effective approach for the treatment of cisplatin-induced ototoxicity (24). The activation of transient receptor potential vanilloid 1 (TRPV1)mediated temporary hearing loss has been shown to occur by initiating an inflammatory process in the cochlea through the activation of NOX3 and STAT1 (25). Corticosteroid therapy reduces inflammation and inhibits apoptosis while activating pro-survival pathways in the organ of Corti following exposure to noise, vibration, cisplatin, aminoglycoside, ischemia/ reperfusion injury, bacterial meningitis and electrode insertion trauma (26). Pro-inflammatory cytokines, such as TNF- $\alpha$, IL- $1 \beta$ and IL-6 play a critical role in cisplatin-induced cochlear 
injury (27). The cochlea, due to its unique anatomical position and isolation, is a closed system, and is therefore unable to flush out the accumulated toxins at the rapid pace of their generation. The accumulated toxins induce the overload of ROS and the dysfunction of the antioxidant system, eventually leading to cell injury and apoptosis $(3,28)$.

It has been demonstrated that cochlear and vestibular functions, such as hearing and balance are related to immune responses $(29,30)$. Although the immune response plays an important role in preventing infectious diseases, such as labyrinthitis in the inner ear, it can also damage the inner ear tissue, causing cochlear degradation and permanent hearing loss (31,32). A previous study demonstrated that cisplatin promoted the production of pro-inflammatory factors involved in the development of ototoxicity in cochlear cells (11). Inflammatory cells are transported to the cochlea through 2 pathways. One involves the infiltration of inflammatory cells into the cochlea from the spiral modiolar vein. The other is that inflammatory cells generated in the endolymphatic sac are directly transferred into the cochlea. Once the inner ear is attacked by bacteria, endotoxins or other inflammatory substances, inflammatory cells, such as monocytes and macrophages migrate to the perilymph and secrete pro-inflammatory cytokines, such as IL-1 $\beta$ and TNF- $\alpha$ during the early phase of inflammation. These pro-inflammatory cytokines induce cochlear spiral ganglionic fibrocytes to produce a variety of inflammatory secretions and mediators that in turn activate inflammatory cells to prolong the inflammatory response in the inner ear (33). So et al verified that the secretion and expression of TNF- $\alpha$, IL- $1 \beta$ and IL- 6 were increased in the cochleae of cisplatin-injected rats and in cultured cochleae (6). Pro-inflammatory factors are directly involved in the process of the cisplatin-induced apoptosis of cochlear cells. The secretion of pro-inflammatory cytokines stimulated by cisplatin in auditory cells in the early phase was unrelated to the change in mRNA expression (6). These data suggest that cisplatin promotes the release of pro-inflammatory factors stored in cells in the early phase and then stimulates the de novo synthesis of pro-inflammatory factors. Pro-inflammatory factor secretion mediated by cisplatin may be the upstream signaling pathway of ROS generation. Pro-inflammatory factor secretion may be responsible for the ototoxicity induced by cisplatin (27). To further confirm this, we measured the levels of IL-1 $\beta$ and IL-6 in the supernatant of cultured basilar membrane and found that cisplatin increased the expression levels of pro-inflammatory factors; the maximum levels were observed at at $12 \mathrm{~h}$ following treatment. These results confirmed that inflammatory responses are involved in cisplatin-induced damage to the cultured basilar membrane.

The increase in the expression levels of STAT6 and p-STAT6 following treatment with cisplatin for $12 \mathrm{~h}$ is in accordance with gene chip analysis, indicating that the expression of STAT6 was significantly enhanced in cochlear cultures following treatment with cisplatin. The IL-1 $\beta$ and IL-6 expression reached maximum levels at $12 \mathrm{~h}$ following treatment with cisplatin. The effects of cisplatin on IL-4, IL- $1 \beta$ and IL- 6 expression were inhibited by TSA. Taken together, cisplatin time-dependently promoted the secretion of inflammatory cytokines in the basilar membrane, and this effect was inhibited by TSA. The level of IL-4 in the supernatant of the cultured basilar membrane reached a maximum at $6 \mathrm{~h}$ following with cisplatin and then decreased rapidly. Thus, IL-4 and the downstream STAT6 signaling cascade play a crucial role in cisplatin-induced ototoxicity.

A previous in vivo study revealed that sodium butyrate provided almost complete protection against cisplatin induced ototoxicity (34). The pathology of the ototoxicity induced by cisplatin is similar to that caused by aminoglycoside antibiotics (35). The application of TSA may rescue cochlear hair cells from aminoglycoside-induced death. TSA suppresses the expression of pro-inflammatory cytokines and inhibits the development of inflammatory diseases, such as ulcerative colitis and rheumatoid arthritis (36). TSA exerts its anti-inflammatory effects by inhibiting the activity of histone deacetylases that aggravate cellular inflammatory responses (36). In the present study, the increase in the expression of pro-inflammatory cytokines and STAT6 and p-STAT6 mediated by cisplatin was significantly decreased by the administration of TSA, demonstrating that TSA exerts a protective effect against cisplatin-induced damage in cochlear hair cells by inhibiting the expression of STAT6 and p-STAT6.

Our findings suggest that TSA protects cochlear hair cells from cisplatin-induced damage by regulating the intracellular levels of histone acetylation, inhibiting the expression of IL-4 and STAT6, and further attenuating excessive inflammatory reactions. In addition, this study provides evidence supporting the novel application of TSA in the pharmacotherapy of cisplatin-induced ototoxicity.

\section{Acknowledgements}

This study was supported by a grant from the National Natural Science Foundation of China (no. 30873131).

\section{References}

1. Dasari S and Tchounwou PB: Cisplatin in cancer therapy: Molecular mechanisms of action. Eur J Pharmacol 740: 364-378, 2014.

2. Barabas K, Milner R, Lurie D and Adin C: Cisplatin: A review of toxicities and therapeutic applications. Vet Comp Oncol 6: 1-18, 2008.

3. Rybak LP, Mukherjea D, Jajoo S and Ramkumar V: Cisplatin ototoxicity and protection: Clinical and experimental studies. Tohoku J Exp Med 219: 177-186, 2009.

4. Sakamoto M, Kaga K and Kamio T: Extended high-frequency ototoxicity induced by the first administration of cisplatin. Otolaryngol Head Neck Surg 122: 828-833, 2000.

5. Rybak LP: Mechanisms of cisplatin ototoxicity and progress in otoprotection. Curr Opin Otolaryngol Head Neck Surg 15: 364-369, 2007.

6. So H, Kim H, Lee JH, Park C, Kim Y, Kim E, Kim JK, Yun KJ, Lee KM, Lee HY, et al: Cisplatin cytotoxicity of auditory cells requires secretions of proinflammatory cytokines via activation of ERK and NF-kappaB. J Assoc Res Otolaryngol 8: 338-355, 2007.

7. Zhou BR, Zhang JA, Zhang Q, Permatasari F, Xu Y, Wu D, Yin ZQ and Luo D: Palmitic acid induces production of proinflammatory cytokines interleukin- 6 , interleukin- $1 \beta$, and tumor necrosis factor- $\alpha$ via a NF- $\kappa \mathrm{B}$-dependent mechanism in HaCaT keratinocytes. Mediators Inflamm 2013: 530429, 2013.

8. Aittomäki S and Pesu M: Therapeutic targeting of the Jak/STAT pathway. Basic Clin Pharmacol Toxicol 114: 18-23, 2014.

9. O'Shea JJ and Plenge R: JAK and STAT signaling molecules in immunoregulation and immune-mediated disease. Immunity 36 : $542-550,2012$

10. Kiu H and Nicholson SE: Biology and significance of the JAK/STAT signalling pathways. Growth Factors 30: 88-106, 2012. 
11. Kim HJ, Oh GS, Lee JH, Lyu AR, Ji HM, Lee SH, Song J, Park SJ, You YO, Sul JD, et al: Cisplatin ototoxicity involves cytokines and STAT6 signaling network. Cell Res 21: 944-956, 2011.

12. Grabiec AM, Tak PP and Reedquist KA: Targeting histone deacetylase activity in rheumatoid arthritis and asthma as prototypes of inflammatory disease: Should we keep our HATs on? Arthritis Res Ther 10: 226, 2008.

13. Chen FQ, Schacht J and Sha SH: Aminoglycoside-induced histone deacetylation and hair cell death in the mouse cochlea. J Neurochem 108: 1226-1236, 2009.

14. Cunningham LL and Brandon CS: Heat shock inhibits both aminoglycoside- and cisplatin-induced sensory hair cell death J Assoc Res Otolaryngol 7: 299-307, 2006.

15. Wang $\mathrm{P}$, Zhang P, Huang J, Li M and Chen X: Trichostatin A protects against cisplatin-induced ototoxicity by regulating expression of genes related to apoptosis and synaptic function. Neurotoxicology 37: 51-62, 2013.

16. Han SB and Lee JK: Anti-inflammatory effect of Trichostatin-A on murine bone marrow-derived macrophages. Arch Pharm Res 32: 613-624, 2009.

17. Kim HJ, Rowe M, Ren M, Hong JS, Chen PS and Chuang DM: Histone deacetylase inhibitors exhibit anti-inflammatory and neuroprotective effects in a rat permanent ischemic model of stroke: multiple mechanisms of action. J Pharmacol Exp Ther 321: 892-901, 2007.

18. Li C, Li X, Miao Y, Wang Q, Jiang W, Xu C, Li J, Han J, Zhang F, Gong B and Xu L: SubpathwayMiner: A software package for flexible identification of pathways. Nucleic Acids Res 37: e131, 2009.

19. Zheng JL and Gao WQ: Differential damage to auditory neurons and hair cells by ototoxins and neuroprotection by specific neurotrophins in rat cochlear organotypic cultures. Eur J Neurosci 8: 1897-1905, 1996

20. Segawa A, Loffredo F, Puxeddu R, Yamashina S, Testa Riva F and Riva A: Exocytosis in human salivary glands visualized by high-resolution scanning electron microscopy. Cell Tissue Res 291: 325-336, 1998

21. Hamers FP, Wijbenga J, Wolters FL, Klis SF, Sluyter S and Smoorenburg GF: Cisplatin ototoxicity involves organ of Corti, stria vascularis and spiral ganglion: Modulation by alphaMSH and ORG 2766. Audiol Neurootol 8: 305-315, 2003.

22. Xiong H, Du W, Zhang YJ, Hong J, Su WY, Tang JT, Wang YC, Lu R, Fang JY and Trichostatin A: Trichostatin A, a histone deacetylase inhibitor, suppresses JAK2/STAT3 signaling via inducing the promoter-associated histone acetylation of SOCS1 and SOCS3 in human colorectal cancer cells. Mol Carcinog 51: 174-184, 2012.

23. Kim J, Cho HJ, Sagong B, Kim SJ, Lee JT, So HS, Lee IK, Kim UK, Lee KY and Choo YS: Alpha-lipoic acid protects against cisplatin-induced ototoxicity via the regulation of MAPKs and proinflammatory cytokines. Biochem Biophys Res Commun 449: 183-189, 2014.
24. Kaur T, Mukherjea D, Sheehan K, Jajoo S, Rybak LP and Ramkumar V: Short interfering RNA against STAT1 attenuates cisplatin-induced ototoxicity in the rat by suppressing inflammation. Cell Death Dis 2: e180, 2011.

25. Mukherjea D, Jajoo S, Sheehan K, Kaur T, Sheth S, Bunch J, Perro C, Rybak LP and Ramkumar V: NOX3 NADPH oxidase couples transient receptor potential vanilloid 1 to signal transducer and activator of transcription 1-mediated inflammation and hearing loss. Antioxid Redox Signal 14: 999-1010, 2011.

26. Abi-Hachem RN, Zine A and Van De Water TR: The injured cochlea as a target for inflammatory processes, initiation of cell death pathways and application of related otoprotectives strategies. Recent Patents CNS Drug Discov 5: 147-163, 2010.

27. So H, Kim H, Kim Y, Kim E, Pae HO, Chung HT, Kim HJ, Kwon KB, Lee KM, Lee HY, et al: Evidence that cisplatininduced auditory damage is attenuated by downregulation of pro-inflammatory cytokines via $\mathrm{Nrf} 2 / \mathrm{HO}-1$. J Assoc Res Otolaryngol 9: 290-306, 2008.

28. Kopke R, Allen KA, Henderson D, Hoffer M, Frenz D and Van de Water T: A radical demise. Toxins and trauma share common pathways in hair cell death. Ann NY Acad Sci 884: 171-191, 1999.

29. Ma C, Billings P, Harris JP and Keithley EM: Characterization of an experimentally induced inner ear immune response. Laryngoscope 110: 451-456, 2000.

30. Rahman MU, Poe DS and Choi HK: Autoimmune vestibulocochlear disorders. Curr Opin Rheumatol 13: 184-189, 2001.

31. Ryan AF, Harris JP and Keithley EM: Immune-mediated hearing loss: Basic mechanisms and options for therapy. Acta Otolaryngol Suppl 122: 38-43, 2002.

32. Stone JH and Francis HW: Immune-mediated inner ear disease. Curr Opin Rheumatol 12: 32-40, 2000.

33. Fujioka M, Kanzaki S, Okano HJ, Masuda M, Ogawa K and Okano H: Proinflammatory cytokines expression in noiseinduced damaged cochlea. J Neurosci Res 83: 575-583, 2006.

34. Drottar M, Liberman MC, Ratan RR and Roberson DW: The histone deacetylase inhibitor sodium butyrate protects against cisplatin-induced hearing loss in guinea pigs. Laryngoscope 116: 292-296, 2006

35. Schacht J, Talaska AE and Rybak LP: Cisplatin and aminoglycoside antibiotics: Hearing loss and its prevention. Anat Rec (Hoboken) 295: 1837-1850, 2012.

36. Toussirot E, Abbas W, Khan KA, Tissot M, Jeudy A, Baud L, Bertolini E, Wendling D and Herbein G: Imbalance between HAT and HDAC activities in the PBMCs of patients with ankylosing spondylitis orrheumatoid arthritis and influence of HDAC inhibitors on TNF alpha production. PLoS One 8: e70939, 2013. 